\title{
Structure Synthesis of Wheelchair-type Lower Limb Rehabilitation System Based on Exercise Rehabilitation
}

\author{
Qiaoling Meng ${ }^{1}$, Meng Wang ${ }^{2}$ and Hongliu $\mathrm{Yu}^{3, *}$ \\ ${ }^{1}$ Shanghai Rehabilitation Equipment Engineering Technology Research Center, Shanghai 200093, China; \\ ${ }^{2}$ Key Laboratory of Neural Function Information and Rehabilitation Engineering of Ministry of Civil Affairs, Shanghai 200093, \\ China \\ ${ }^{3}$ Institute of Rehabilitation Engineering \& Technology, School of Medical Instrument and Food Engineering, University of \\ Shanghai for Science and Technology, Shanghai 200093, China \\ ${ }^{*}$ Corresponding author
}

\begin{abstract}
This paper presents a new way of structure composition of wheelchair-style lower limb rehabilitation mechanisms to develop a systematic and modularized structure synthesis methodology of exercise rehabilitation mechanisms based on exercise rehabilitation. First, the movement principle of hip and knee joints under exercise rehabilitation on chair is deduced in order to provide structure formulation. Then, two structures are researched according to the proposed principle. Further, the simulation experiments for the structures have been done to verify the proposed approach and the experimental results show that the average cumulative error of hip joint is $4.43 \%$ and the average cumulative error of knee joint is $0.01 \%$.
\end{abstract}

Keywords-exercise rehabilitation of lower limb; physical therapy; hip-knee linkage; size synthesis

\section{INTRODUCTION}

$75 \%$ to $80 \%$ of stroke patients are troubled by sequelae such as hemiplegia [1]. Physical function exercise rehabilitation is one of the main ways to help the rehabilitation of the affected side movement and nerve function. Rehabilitation of stroke patients is divided into acute phase, recovery phase, and sequelae phase. In the acute phase and recovery phase, a large number of repetitive exercise training or activities for daily living activities are required. The exercise rehabilitation robot is one of the ways to solve the problem of low training efficiency caused by long exercise rehabilitation cycle [2].

At present, there are many kinds of lower limb rehabilitation training robots, and their configuration methods are also different. Passive hip-knee joint movement is mainly achieved through the mechanism to complete passive rehabilitation training. Beomsoo et al. used the wheelchair as a weight reduction and support system according to the characteristics of wheelchair patients for rehabilitation [3-4]. The exoskeleton structure was used to design a wheelchair-based hip-knee joint lower limb exercise rehabilitation system to ensure weight loss and safety. But the system has a complex structure and a large rack, which is not suitable for moving and training in a narrow space. Shanghai Jiaotong University's Yin Yuehong team designed a bed-type lower extremity exoskeleton rehabilitation robot that can perform hip-knee-ankle joint training combined with human bionics principles [5]. However, it is not convenient for patients to move daily with the bed body as a platform, and the patients need to transfer to the bed body when training. Yan Hua, Zhejiang University, combined with clinical experience to design a wheelchair-type lower limb exercise rehabilitation exoskeleton system for hip-knee motion, but the product is inefficient in exercise rehabilitation, heavy-duty and complicated in operation [6].

From this, the existing training robot for lower limb rehabilitation mainly simulates the normal gait and skeletal system of the human body and designs it with the technique of exoskeleton. However, the gait of hemiplegia patients is very different, and the lower limb posture and gait are different from normal people, and they can't realize the normal movement rule accurately during training. Therefore, it is necessary to study the proper configuration for guiding the design of lower limb rehabilitation robots. In addition, most stroke patients use wheelchairs as their main mobile tools, and the transfer of robots from wheelchairs to lower limb exercise rehabilitation is one of the difficult problems of lower limb exercise rehabilitation. Therefore, this paper proposes a comprehensive method for simulating the configuration of physiotherapy to solve the problem of poor adaptability and low training efficiency of lower limb rehabilitation robots. First of all, it studies the methods of physical rehabilitation of lower extremities to explore the characteristics of physical therapies of rehabilitation therapists in maintaining the motion of lower extremities. Then the physical rehabilitation therapy is mathematically modeled, and then combined with the rehabilitation method to propose a configuration of a 2-DOF wheelchair-type lower limb rehabilitation training robot that meets the needs of patients with lower limb dysfunction.

\section{The Movement PRINCIPLE OF HIP AND KNEE JOINTS BASED ON EXERCISE REHABILITATION ON CHAIR}

Physical exercise rehabilitation refers to the training methods used by freehanders for patients whose activities are limited due to the causes of paralysis and pain, and who are unable to perform autonomous exercise or assisted active exercise [7]. The lower limbs of the human body are formed 
by the hip joints, knee joints and ankle joints. The hip joint and the ankle joint are used as spherical joints and the knee joint is used as an ordinary hinge joint. Therefore, the simplified structure of the human lower limb skeleton in the sitting posture can be obtained, as shown in Figure I. The hip movement angle is $\alpha$, and the knee movement angle is $\beta$.

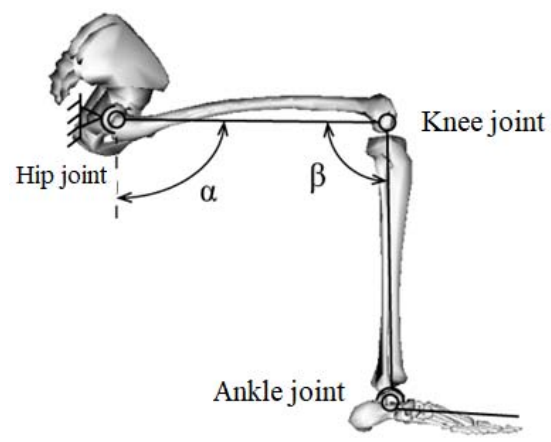

FIGURE I. SIMPLIFICATION OF HUMAN LOWER LIMB BONE STRUCTURE

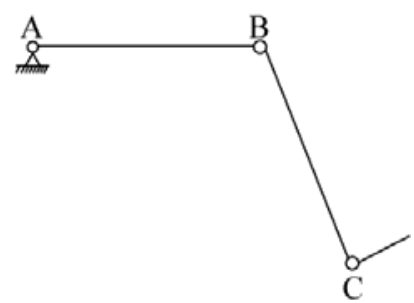

FIGURE II. LOWER LIMB JOINT MOBILITY TRAINING MECHANISM

Assume that the rehabilitation therapist's training operation is uniform. The hip initial angle is $b_{1}$, and the knee initial angle is $b_{2}$. The time of stage 1 is $t_{1}$, the time of Stage 2 is $t_{2}$, the time of Stage 3 is $t_{3}$, the time of Stage 4 is $t_{4}$. Assume that the hip joint movement speed is $\mathrm{k}_{1}$, the knee joint movement speed is $\mathrm{k}_{2}$, and one training cycle time is $\mathrm{t}_{1}+\mathrm{t}_{2}+\mathrm{t}_{3}+\mathrm{t}_{4}$.

Stage one, hip flexion:

$$
\alpha=k_{1} t+b_{1} \quad \beta=b_{2}
$$

Stage two, knee extension:

$$
\alpha=k_{1} t_{1}+b_{1} \quad \beta=k_{2}\left(t-t_{1}\right)+b_{2}
$$

Stage three, knee flexion:

$$
\alpha=k_{1} t_{1}+b_{1} \quad \beta=-k_{2}\left(t-t_{1}-t_{2}\right)+b_{2}+k_{2} t_{2}
$$

Stage four, hip extension:

$$
\alpha=k_{1} t_{1}+b_{1}-k_{1}\left(t-t_{1}-t_{2}-t_{3}\right) \quad \beta=b_{2}
$$

\section{Structure SyntheSIS OF WHEELCHAIR-STYLE LOWER LIMB REHABILITATION MECHANISMS}

The study of lower limb exercise rehabilitation equipment is based on the exoskeleton of the human lower limbs. According to the characteristics of the lower limbs of the human body, through the analysis of the therapist's training on the maintenance of hip-knee joint motion in the sitting posture it can be known that when the therapist performs rehabilitation treatment, the hip-knee joint of the patient is a linkage relationship. In order to maintain the training effect of the joint motion of the lower extremity, this design limits the remaining degrees of freedom of the hip joint and only retains the flexion and extension, which is simplified as a hinge joint. The simplified 2-DOF lower limb joint motion training mechanism is shown in Figure II. A and B are hinge links, and C is a special hinge mechanism that can adjust the angle. The $\mathrm{AB}$ segment is the thigh segment, the $\mathrm{BC}$ segment is the calf segment, and the distal segment is the sole of the foot.

\section{A. Structure Synthesis of Wheelchair-Style Rehabilitation Mechanism Based on Exercise Rehabilitation Principle}

The subject's lower limb joint mobility training institutions studied in this study have two ways to provide power. The Configuration 1 is powered by two pusher motors, which is a full drive system (as shown in Figure III.). The full-drive system can fully control the training angle of the hip-knee joint, maximally approaching the therapist's rehabilitation treatment. The Configuration 2 is a split full drive system, which is powered by two pusher motors to control the motion of the hip and knee joints. (as shown in Figure IV.). The hip-knee joint of configuration 2 has no effect on each other, so the control is simpler and it is easier to achieve the therapist's operating technique.

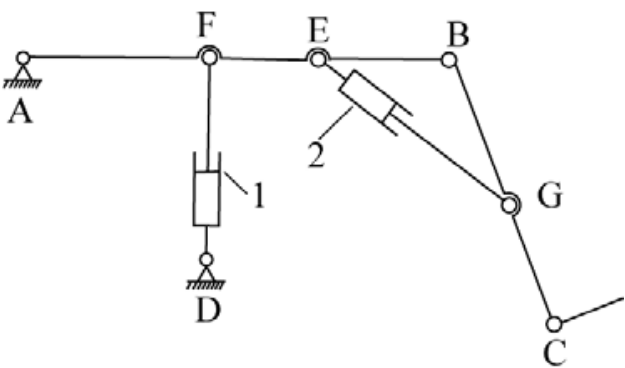

FIGURE III. CONFIGURATION 1

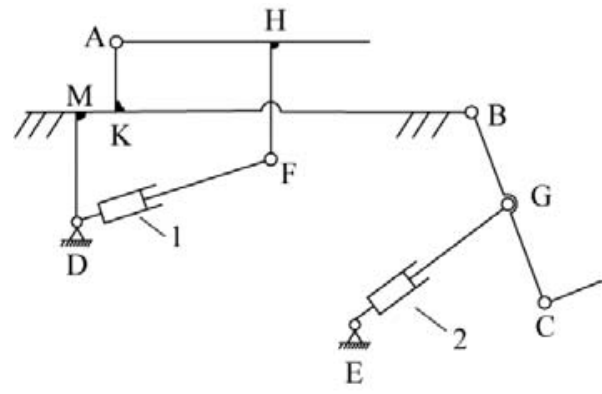

FIGURE IV. CONFIGURATION 2

Because of adopting the same design method, this article only establishes a mathematical model of the configuration 1, 
and analyzes the movement law during hip-knee joint training. Then this article compares it with the therapist's manipulation of hip-knee joint rehabilitation and further optimizes the design of the mechanism to achieve similar results as the therapist's rehabilitation.

\section{B. Size Synthesis of Wheelchair-Style Rehabilitation Mechanism Based on Exercise Rehabilitation Principle}

For the optimal design of the Configuration 1, the size of the hip joint $\alpha$ is obtained from the dimensions of the rod AF, the rod $\mathrm{AD}$, and the push rod motor $\mathrm{DF}$, and then the size $\beta$ of the knee joint is calculated. Assume that the lengths of the rod $\mathrm{AF}$, the distance $\mathrm{AD}$, the $\operatorname{rod} \mathrm{BE}$, and the rod $\mathrm{BG}$ are $1_{1}, 1_{2}, 1_{3}, 1_{4}$, the length of the push rod motor 1 is $d_{1}$, and the length of the push rod motor 2 is $d_{2}$. The hypothesis method is adopted to simplify the hip and knee joint activity maintenance training mechanism as a turning guide rod mechanism, and the simplified rotation guide rod mechanism is shown in FIGURE $\mathrm{V}$. The hip joint angle (the angle between the thigh and the horizontal position) is $\alpha$, and the knee joint (the angle between the thigh and the lower leg) is $\beta . \mathrm{d}_{1}=\mathrm{V}_{1} \mathrm{t}, \mathrm{d}_{2}=\mathrm{V}_{2} \mathrm{t},\left(\mathrm{V}_{1}, \mathrm{~V}_{2}\right.$ are the speed of the push rod motor 1,2 respectively, $t$ is the time).

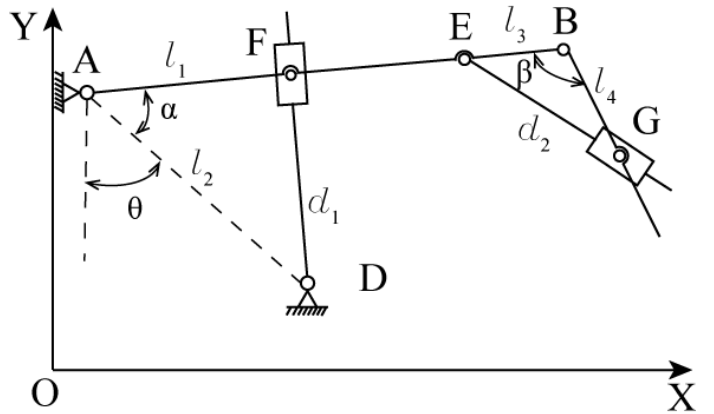

FIGURE V. CONFIGURATION 1 MATHEMATICAL COORDINATE SYSTEM

From the coordinate system X-O-Y in Figure V:

$$
\cos \alpha=\frac{l_{1}^{2}+l_{2}{ }^{2}-d_{1}{ }^{2}}{2 l_{1} l_{2}} \quad \cos \beta=\frac{l_{3}^{2}+l_{4}{ }^{2}-d_{2}{ }^{2}}{2 l_{3} l_{4}}
$$

Design variables are:

$$
x=\left[l_{1}, l_{2}, d_{1 \text { min }}, d_{1 \text { max }}, l_{3}, l_{4}, d_{2 \min }, d_{2 \max }\right]
$$

$\theta+\alpha, \beta$ is the angle of hip joint movement when using the lower limb exercise rehabilitation mechanism and the therapist's rehabilitation treatment. When the difference between $\alpha^{\prime}$ and $\beta^{\prime}$ is 0 , the two are exactly the same, and the sum of the two minimum differences is the optimization function, ie:

$$
f(x)=\sum_{i=1}^{n} \sqrt{\left(\alpha-\alpha^{\prime}\right)^{2}+\left(\beta-\beta^{\prime}\right)^{2}}
$$

The constraints are:

a) The crank is larger than the rack length, ie:

$$
g 1(x): l_{1}>l_{2} \quad g 2(x): l_{4}>l_{3}
$$

b) According to the body size regulations $l \_1$ is not greater than $500 \mathrm{~mm}$ and $1 \_4$ is not greater than $400 \mathrm{~mm}$, ie:

$$
g 3(x): l_{1}<500 \quad g 4(x): l_{4}<400
$$

c) In order to ensure good transmission of the mechanism, select a minimum transmission angle greater than $45^{\circ}$, ie:

$$
g 5(x): \arccos \frac{l_{1}^{2}+d_{1}{ }^{2}-l_{2}^{2}}{2 l_{1} d_{1}} \geq \frac{\pi}{4} \quad g 6(x): \arccos \frac{l_{4}^{2}+d_{2}^{2}-l_{3}^{2}}{2 l_{4} d_{2}} \geq \frac{\pi}{4}
$$

d) In order to ensure the safety of rehabilitation, combined with clinical data, the hip joint angle is between $90^{\circ}$ and $130^{\circ}$, and the knee joint angle is between $90^{\circ}$ and $120^{\circ}$, ie:

$$
\begin{array}{ll}
g 7(x): \theta+\arccos \frac{l_{1}^{2}+l_{2}{ }^{2}-d_{1}{ }^{2}}{2 l_{1} l_{2}} \geq \frac{\pi}{2} & g 8(x): \theta+\arccos \frac{l_{1}^{2}+l_{2}{ }^{2}-d_{1}{ }^{2}}{2 l_{1} l_{2}} \leq \frac{3 \pi}{4} \\
g 9(x): \arccos \frac{l_{3}{ }^{2}+l_{4}{ }^{2}-d_{2}{ }^{2}}{2 l_{3} l_{4}} \geq \frac{\pi}{2} & g 10(x): \arccos \frac{l_{3}{ }^{2}+l_{4}{ }^{2}-d_{2}{ }^{2}}{2 l_{3} l_{4}} \leq \frac{7 \pi}{9}
\end{array}
$$

The operating method of the therapist in physical therapy under the sitting posture was selected as the boundary condition for the robot to realize the rehabilitation function. This article selects the therapist's operation of hip-knee joints within 90 seconds as an example. The hip movement angle is from $90^{\circ}$ to $135^{\circ}$, and the knee movement angle is from $90^{\circ}$ to $140^{\circ}$. In this paper, the mathematical model of the operation method is fitted and optimized. The optimization results are shown in Table I., Table II.

\section{TABLE I. HIP JOINT OPTIMIZATION SOLUTION RESULTS OF CONFIGURATION 1}

\begin{tabular}{cccc}
\hline$l_{1} / \mathbf{m m}$ & $l_{2} / \mathbf{m m}$ & $d_{1 \min } / \mathbf{m m}$ & $d_{1 \max } / \mathbf{m m}$ \\
\hline 220.18 & 214.50 & 298.21 & 387.92 \\
\hline \multicolumn{4}{c}{ TABLE II. KNEE JOINT OPTIMIZATION RESULTS OF } \\
CONFIGURATION 1 \\
\hline$l_{3} / \mathbf{m m}$ & $l_{4} / \mathbf{m m}$ & $d_{2 \min } / \mathbf{m m}$ & $d_{2 \max } / \mathbf{m m}$ \\
\hline 87.14 & 87.14 & 123.23 & 164.07 \\
\hline
\end{tabular}

\section{SIMULATION EXPERIMENTS}

According to the results in Table I and Table II., the hip joint training movement angle is $91.62^{\circ} \sim 131.34^{\circ}$, and the knee joint training movement angle is $90.00^{\circ} \sim 140.58^{\circ}$. According to the optimization results and the principle of physical therapy, a corresponding mathematical model was established to obtain a comparison curve between the theoretical results and physical therapy (as shown in FIGURE VI.). The simulation experiment shows that the sum of the errors of the hip joints $\mathrm{fl}(\mathrm{x})$ is 0.0443 , and the sum of the errors of the knee 
joint mechanisms $\mathrm{f} 2(\mathrm{x})$ is 0.0010 .

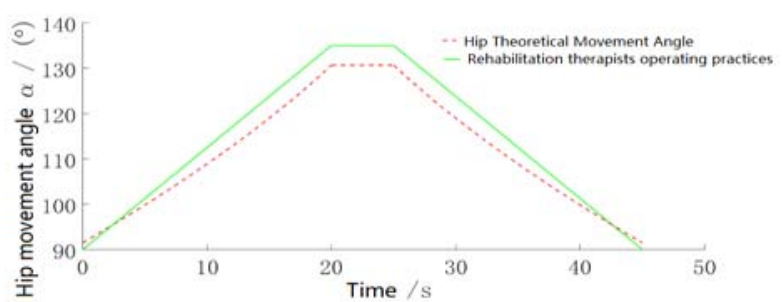

(A) SIMULATION EXPERIMENT OF HIP JOINT

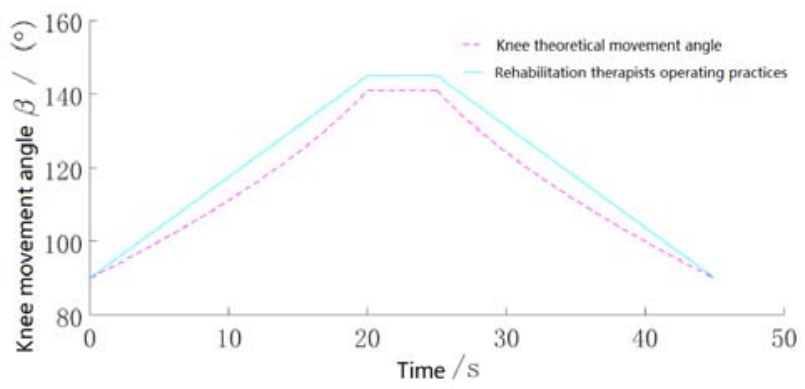

(B) SIMULATION EXPERIMENT OF KNEE JOINT

FIGURE VI. HIP-KNEE JOINT THEORETICAL PLANNING EXERCISE ANGLE OF CONFIGURATION 1 COMPARED WITH THERAPIST'S OPERATING TECHNIQUES

\section{CONCLUSION}

This paper presents a systematic structure synthesis method of wheelchair-style lower limb rehabilitation mechanisms based on hip-knee joint movement principle in exercise rehabilitation. Two structures are built and analyzed. In the end, the simulation experiments for the structures have been done to verify the proposed approach. The experimental results show that the average cumulative error of hip joint is $4.43 \%$ and the average cumulative error of knee joint is $0.01 \%$.

\section{ACKNOWLEDGMENTS}

The program was granted by Shanghai science and technology support project, number: 5DZ1941902 \& 16441905202 and Shanghai Engineering Research Center of Assistive Devices, number: 15DZ2251700.

\section{REFERENCES}

[1] Zhou Peiyi, Wu Ziqiang, Xie Zhiquan, etc. Research progress on risk factors of stroke [J]. Chinese Journal of Gerontology, 2012, 32(16):3590-3594.

[2] Riener R, Nef T, Colombo G. Robot-aided neurorehabilitation of the upper extremities [J]. Medical\&Biological Engineering\&Computing, 2005, 43(1):2-10.

[3] Hwang B, Jeon D. Development and preliminary testing of a novel wheelchair integrated exercise/ rehabilitation system[C].// IEEE International Conference on Rehabilitation Robotics. London: IOS Press, 2013:1-6.

[4] Hwang B, Jeon D. A wheelchair integrated lower limb exercise/ rehabilitation system: Design and experimental results on the knee joint[C].// IEEE/SICE International Symposium on System Integration. 2012:164-169.

[5] Sun Hongwei. A study on the rehabilitation robot of the lower limb exoskeleton[D]. Shanghai Jiao Tong university, 2012.
[6] Yan Hua, Yang can Jun. Study on the outer skeleton system of training for lower limb movement rehabilitation [C]// national symposium on rehabilitation medical engineering and rehabilitation engineering. 2010.

[7] Yu Duisheng. Exercise therapy and exercise therapy [M]. Huaxia publishing house, 2002. 